\title{
Conflicto de valores en el sistema de salud de Colombia: entre la economía de mercado y la normativa constitucional, 2007-2009
}

\author{
Gloria Molina y Andrés Ramírez²
}

Forma de citar

Molina G, Ramírez A. Conflicto de valores en el sistema de salud de Colombia: entre la economía de mercado y la normativa constitucional, 2007-2009. Rev Panam Salud Publica. 2013;33(4):287-93.

RESUMEN Objetivo. Presentar los aspectos morales en conflicto en los procesos de toma de decisiones clinico-administrativas dentro del Sistema General de Seguridad Social en Salud (SGSSS) de Colombia.

Métodos. La investigación se llevó a cabo entre 2007 y 2009 en seis ciudades colombianas (Barranquilla, Bogotá, Bucaramanga, Leticia, Medellín y Pasto), según una metodología cualitativa basada en la teoría fundamentada. Se realizaron 179 entrevistas en profundidad a médicos, personal de enfermería y administradores con amplia experiencia en el sector de la salud, y 10 grupos focales con usuarios y líderes de organizaciones comunitarias vinculadas a la salud. Las entrevistas, con preguntas semiestructuradas y según una guía elaborada previamente, recababan información personal y profesional.

Resultados. En el proceso de toma de decisiones en la atención en salud en Colombia se identificaron elementos de dos morales enfrentadas: la rentista, caracterizada por la axiología neoliberal de la economía de mercado (práctica) y otra fundamentada en la axiología constitucional (normativa) del Estado social de derecho. Predomina el enfoque utilitarista e individualista y se promueve el lucro individual y empresarial por encima del interés colectivo; esto favorece prácticas que contravienen los derechos de las personas y la comunidad.

Conclusiones. El predominio de la moral que responde al modelo de mercado en el SGSSS colombiano genera condiciones adversas a los principios y valores que deben orientar el sistema de salud como garante del derecho a la salud y la dignidad humana. Para la toma de decisiones en salud es necesario tener en cuenta, además de los elementos técnicos y científicos, los principios y valores que están enfrentados y reflexionar sobre ellos para su legitimación.

Palabras clave Sistemas de salud; moral; ética; privatización; Colombia.

La moral tiene un sustento social y se construye en los procesos de interacción y socialización $(1,2)$, en los cuales el individuo internaliza un conjunto de valores, prácticas y roles que moldean la construcción de su identidad social e individual. Pero en las sociedades existen diferentes

\footnotetext{
1 Facultad Nacional de Salud Pública, Universidad de Antioquia, Medellín, Colombia. La correspondencia se debe dirigir a Gloria Molina. Correo electrónico: molinag@saludpublica.udea.edu.co

2 Universidad Santo Tomás, Medellín, Colombia.
}

referentes axiológicos que conforman diversas morales en pugna constante por legitimación, ${ }^{3}$ lo que impulsa cambios de identidad individual y colectiva, generadores de transformación social (3).

En el campo de la filosofía, la ética corresponde a la reflexión sobre la moral

\footnotetext{
3 Fields B. Human rights theory: criteria, boundaries, and complexities. Fifth International Congress of Qualitative Inquiry; 2009 May 20-23. University of Illinois, Urbana-Champaign, Illinois; 2009.
}

(4) y proporciona elementos para analizar las condiciones en las que las normas y los valores morales sean imperativos y vinculantes (1).

La moral detrás del sistema de seguridad social en salud de Colombia

Las teorías utilitaristas han influido en diversos campos, incluidos los sistemas de salud. Las corrientes utilitaristas propugnan que la distribución genere la máxima 
satisfacción al mayor número de personas al menor costo posible. En las reformas de salud, el costo-beneficio y la identificación de prioridades según las pérdidas y las ganancias se convirtieron en la mejor expresión de esta posición. Uno de los más claros ejemplos de la aplicación de las teorías utilitaristas en los sistemas de salud es, probablemente, la reforma de la seguridad social en Colombia (5).

El Sistema General de Seguridad Social en Salud (SGSSS) de Colombia, establecido según la Ley 100 de 1993 (6), tiene su fundamentación en directrices neoliberales internacionales justificadas en la búsqueda de mejores coberturas, acceso, calidad, equidad, eficiencia y sostenibilidad financiera del sistema de salud $(7,8)$. Esta ley estableció el aseguramiento como medio para acceder a los servicios y creó las empresas promotoras de salud (EPS) públicas y privadas, responsables de proveer a sus afiliados un plan obligatorio de salud, por lo cual reciben el denominado pago por capitación, es decir, una suma de dinero por persona afiliada. Esta ley también cambió el subsidio de la oferta por el subsidio de la demanda, introdujo el mercado y la competencia entre las EPS y las instituciones prestadoras de servicios (IPS) públicas y privadas, redujo el papel desempeñado por el Estado y amplió la participación privada. Sin embargo, en el SGSSS se ha promovido un nivel de utilitarismo que le ha impedido alcanzar sus metas de equidad, accesibilidad y calidad. Aunque se ha logrado asegurar un mayor porcentaje de la población $(87,2 \%$ en 2010) y la participación privada ha aumentado (9), no se ha logrado un mayor acceso efectivo a los servicios de salud debido a múltiples barreras administrativas, geográficas y económicas generadas, principalmente, por las EPS (10-14). Aunque algunas EPS privadas se han posicionado entre las empresas más rentables del país -como SaludCoop, Salud Total y Colsanitas- (15, 16), han aumentado las quejas y tutelas por la negación sistemática de servicios a los usuarios. Según la Defensoría del Pueblo (17), en 2011 se presentaron 105945 tutelas. Además, ha habido informes de que algunas EPS ofrecen condiciones laborales insatisfactorias, tienen asimetría de información, segmentan y seleccionan la población con menos riesgos en salud, provocan conflictos interinstitucionales por no pagar oportunamente a las IPS los servicios prestados a sus afiliados y se han detectado casos de corrupción (18). A pesar del incremento en la oferta de servicios, principalmente privada, persisten inequidades y desigualdad en la distribución por regiones (19-22).

\section{La moral que propugna la Constitución Política de Colombia}

A diferencia de la moral del sistema económico neoliberal, la Constitución Política de Colombia de 1991 (23) se fundamenta en los valores y principios propios de un Estado social de derecho. Al respecto, Otero afirma que los valores son, en definitiva, los cimientos sobre los que la sociedad quiere que se establezcan las leyes para garantizar la satisfacción de las necesidades sociales y proporcionar el bienestar y el bien común (24). Los principios y valores constitucionales buscan racionalizar y humanizar el sistema normativo en general -y el sistema de salud en particular - para lograr un orden social más justo. Así, según Rawls, "el principio para la sociedad es promover tanto como sea posible el bienestar del grupo" (25), lo que constitucionalmente está consagrado como la prevalencia del interés general (artículo 1. ${ }^{\circ}$ de la Constitución Política). De esa forma, el sistema económico en general y el SGSSS en particular deben reconocer y cumplir principios y valores tales como la dignidad humana, la autonomía, la beneficencia y no maleficencia, la responsabilidad, la honestidad, la solidaridad, la cooperación y la confianza, entre otros.

El objetivo de este artículo, derivado de un estudio más amplio que analizó los elementos que inciden en las decisiones relacionadas con la atención en salud (19), es presentar los aspectos morales en conflicto en los procesos de toma de decisiones clínico-administrativas dentro del SGSSS colombiano.

\section{MATERIALES Y MÉTODOS}

La investigación se llevó a cabo entre 2007 y 2009 en seis ciudades colombianas: Barranquilla, Bogotá, Bucaramanga, Leticia, Medellín y Pasto, las cuales tienen diferentes características socioeconómicas, políticas, culturales e institucionales. Se adoptó una metodología de investigación cualitativa (26) basada en la teoría fundamentada (27), cuyo soporte teórico y filosófico es el interaccionismo simbólico.
Se entrevistaron profesionales de la salud (médicos, personal de enfermería y administradores) seleccionados por tener al menos 10 años de experiencia, sus conocimientos del sistema de salud y su vinculación a la atención de pacientes y labores administrativas o de dirección en instituciones aseguradoras o prestadoras de servicios de salud, ya sean públicas o privadas. Se entrevistó a 179 profesionales (29 por ciudad como promedio), de ellos $48 \%$ laboraban en entidades públicas y $52 \%$ en privadas. También se establecieron 10 grupos focales (28) con usuarios vinculados por lo menos 5 años a estos servicios y representantes de organizaciones comunitarias en salud. Las entrevistas, con preguntas semiestructuradas y según una guía elaborada previamente, recababan información personal (como edad, sexo, profesión, escolaridad, años de experiencia) y profesionales (elementos que intervienen en las decisiones sobre la prestación de servicios de salud; decisiones profesionales en relación con las normas del SGSSS, el acceso y la calidad de los servicios; implicaciones éticas de las decisiones profesionales; dimensiones axiológicas en la práctica del SGSSS).

Las entrevistas se grabaron, transcribieron y analizaron mediante la codificación y la categorización sistemática (abierta, axial y selectiva), según el método de la teoría fundamentada. Los resultados se validaron en foros realizados en cada ciudad, con la participación de los entrevistados, profesionales del sector de la salud, académicos y representantes de organizaciones comunitarias, para verificar si los resultados representaban la realidad vivida y percibida por la comunidad, sin sesgos introducidos por la perspectiva de los investigadores $(29,30)$.

Para el análisis se usaron los paquetes informáticos Microsoft Word, Microsoft Excel, Atlas.ti y CmapTools versión 4.16.

A cada entrevistado se le informó sobre los objetivos del estudio, se le solicitó el consentimiento informado y se le garantizó la confidencialidad de su identidad y de la institución para la cual trabajaba.

\section{RESULTADOS}

A pesar de las diferencias socioeconómicas, políticas e institucionales existentes entre las ciudades abarcadas por el estudio, los procesos de toma de decisiones en la atención en salud no difirieron entre sí, posiblemente debido a que las 
directrices nacionales de las aseguradoras privadas y las entidades estatales son obligatorias para todo el país.

Del análisis de las entrevistas individuales y de grupo realizadas se generaron 11976 códigos (comentarios críticos), agrupados en 74 categorías descriptivas, y emergieron 13 categorías analíticas (figura 1). El contenido de estas categorías se describe en una publicación previa (19), por lo que a continuación se analizan solamente los aspectos éticos de los procesos de toma de decisiones relacionadas con los servicios de salud.

En el análisis se identificaron elementos de dos morales enfrentadas: la rentista, caracterizada por la axiología neoliberal de la economía de mercado (práctica), que enfatiza el utilitarismo, y otra que se fundamenta en la axiología constitucional (normativa) del Estado social de derecho.

\section{Estrategias de gestión que afectan a la calidad de la atención en salud}

La concepción rentista está basada en las leyes del mercado y la competencia y busca garantizar la rentabilidad financiera mediante la gestión institucional. Con esto se imponen barreras administrativas, geográficas y económicas al acceso y se controla la demanda de los servicios. Así lo reflejan los siguientes comentarios de profesionales clínicos, personal administrativo y usuarios: ${ }^{4}$

"Ya no importa la vida del paciente, ni la calidad de vida, sino el dinero" (clínico, 35-7).

"Yo no tuve ningún gerente de EPS que me preguntara cuáles son mis tasas de mortalidad y mis tasas de infecciones, sino cuál es mi tarifa" (clínico, 36-9).

La moral del SGSSS privilegia la productividad y la eficiencia y se esfuerza por incrementar el volumen de actividades, aunque sin tomar en cuenta su calidad. Por ejemplo, se disminuye el tiempo de consulta para producir más con los mismos recursos humanos. El siguiente comentario ilustra esta afirmación:

“Algunas EPS hacen llamado de atención cuando el médico demora más tiempo con el paciente" (clínico, 47-6).

\footnotetext{
4 Los comentarios, que se presentan para ilustrar algunos de los hallazgos, corresponden a afirmaciones hechas por los entrevistados.
}

FIGURA 1. Categorías analíticas relacionadas con las decisiones en la atención sanitaria, el acceso y la calidad de los servicios ${ }^{a}$

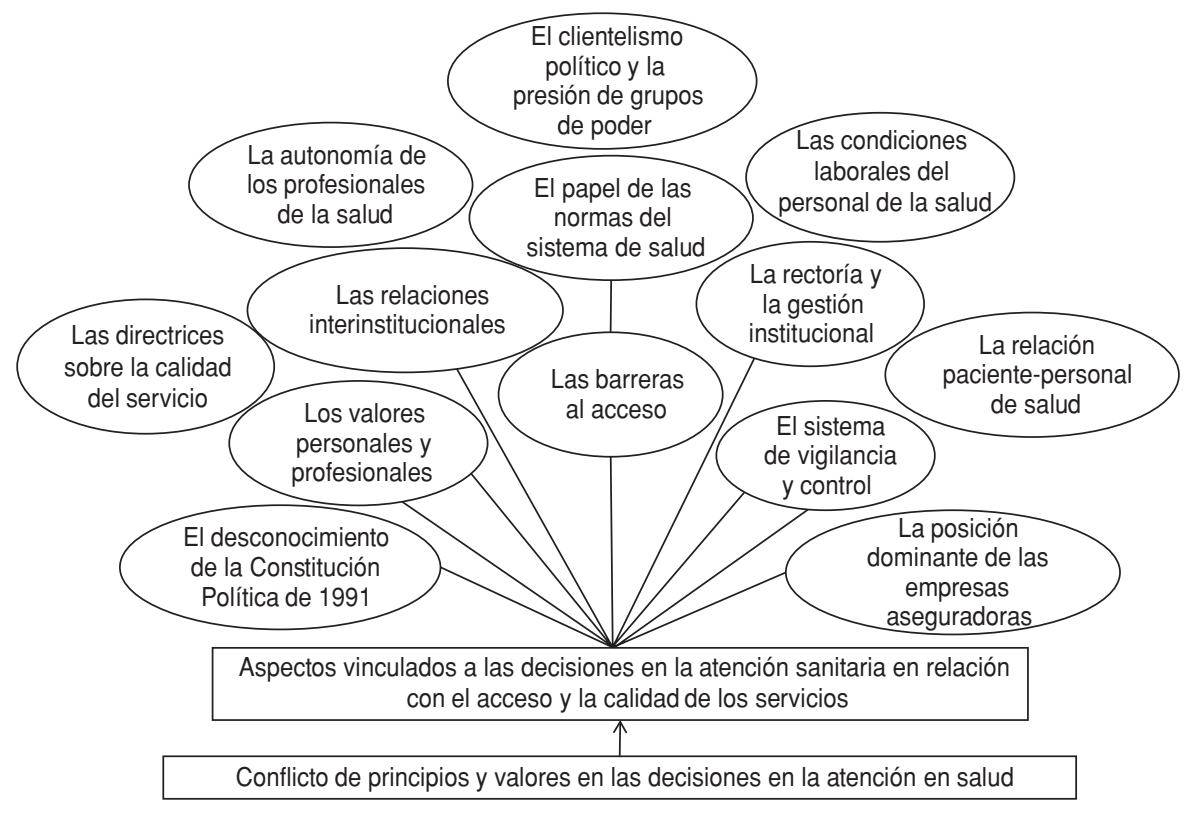

a El contenido de estas categorías se describe en una publicación previa (19).

Las EPS, con una concepción rentista, niegan frecuentemente el pago de algunos servicios, lo que amenaza el funcionamiento de las IPS, ocasiona conflictos interinstitucionales y limita el acceso. Así, la accesibilidad queda condicionada a la reputación de la EPS como pagador y los servicios se asignan según esa característica, y no según la necesidad del paciente.

“Las EPS retienen el pago de los servicios prestados por las IPS, con fines lucrativos" (administrativo, 97-8).

La fragmentación de la atención tiene su origen en los procesos de contratación de los servicios basados en bajos precios. Las aseguradoras contratan servicios de manera dispersa y fragmentada con múltiples instituciones, distantes del lugar de trabajo o la vivienda del afiliado, lo que genera barreras geográficas $y$ mayores desplazamientos y costos de transporte. Esto obliga a los pacientes a buscar atención y autorizaciones en varias instituciones; por ejemplo, los pacientes con cáncer tienen que pedir autorizaciones reiteradamente antes de cada sesión de quimioterapia o radioterapia.

“El fraccionamiento de la atención tiene objetivos rentistas claros, de corto plazo, sin importar las complicaciones clínicas que se generen" (clínico, 76-3).

“Los padres de los niños con cáncer tienen que atravesar varias veces la ciudad para lograr una autorización" (usuario, 105-11).

\section{Estrategias de gestión que restringen los principios de autonomía, beneficencia y no maleficencia}

Otras manifestaciones de la gestión institucional también afectan negativamente a los pacientes y menoscaban los principios de autonomía, beneficencia y no maleficencia, por ejemplo, la coacción a la libre prescripción médica en aras de minimizar los costos mediante la imposición de guías de práctica clínica elaboradas por las aseguradoras. Estas guías indican, entre otros puntos, que algunos cuidados propios de enfermería se supediten a prescripciones médicas, lo que perjudica la atención.

Además, se trata de reducir los costos laborales mediante la contratación de profesionales inexpertos a corto plazo - sin seguridad social y a través de terceros-, a los que se les condiciona la renovación del contrato a la obediencia de las normas institucionales dirigidas a lograr metas financieras. Los médicos 
con mayor número de prescripciones pueden ver amenazadas las posibilidades de renovar sus contratos.

Algunas IPS cobran al personal de salud los servicios prestados a los pacientes que la EPS se negó a pagar, lo que coarta la libertad de decisión clínica de estos profesionales.

Algunos comentarios ilustran estas afirmaciones:

\footnotetext{
"Aquí a los médicos jóvenes los contratan a destajo: «usted venga y trabájeme 15 días y aquí tiene su plata», no les interesa si tienen protección social o no" (clínico, 111-6).
}

\begin{abstract}
“La autonomía está muy restringida por esos controles que se imponen y los estímulos perversos; al que no formula medicamentos ni ordena exámenes, lo premian y estimulan" (clínico, 105-7).
\end{abstract}

“Nos reúnen a un grupo de médicos y nos dicen: está muy alto el costo médico, hay que disminuirlo. No hay que operar a todos los pacientes, hay que diferirlos y darles menos medicamentos" (clínico, 78-5).

\section{Manifestaciones que violentan los principios de honestidad, responsabilidad, confianza y solidaridad}

En algunas instituciones, tanto públicas como privadas, los partidos políticos y grupos de poder tienen una palpable presencia, lo que les da un mayor acceso a los recursos públicos de salud. En algunos hospitales públicos, las áreas hospitalarias están distribuidas entre los partidos políticos, lo que afecta a la vinculación del personal. Este clientelismo se ha favorecido con la descentralización y la privatización; el vínculo entre las aseguradoras, las élites financieras y los partidos políticos; el insuficiente nivel de rectoría, asistencia técnica, vigilancia y control; así como con la inestabilidad laboral. Por otro lado, el utilitarismo individualista propicia algunas prácticas de los diferentes actores $-\mathrm{y}$ entre ellos mismos- que socaban los principios de honestidad, confianza y responsabilidad. Entre esas prácticas se encuentran:

- la manipulación de las bases de datos de afiliados y la asimetría de la información con fines rentistas

- la contratación de personal no calificado
- el aumento ficticio del número de afiliados para recibir más ingresos

- la entrega de incentivos a los profesionales para que nieguen servicios a los pacientes

- las prescripciones innecesarias para incrementar la facturación

- la limitación del flujo de recursos, especialmente de las EPS y el gobierno, que buscan obtener el máximo beneficio económico al demorar los pagos a las IPS.

Algunos de los comentarios que respaldan estas afirmaciones provienen también de profesionales clínicos, como los siguientes: "Se contrata personas con impedimentos
legales" (clínico, 154-10).

"Perversión en el sistema: algunas EPS falsifican firmas para mostrar 'carnetización' de la población" (clínico, 81-1).

"El Estado se comporta como un mal pagador de los servicios que contrata" (clínico, 115-9).

\section{DISCUSIÓN}

Los resultados muestran que en el sistema de salud colombiano existe una confrontación entre la moral auspiciada por el mercado y la moral que dicta la constitución, lo que genera conflictos de valores en la toma de decisiones. $\mathrm{La}$ moral del mercado se ha manifestado con fuerza e impregna y condiciona decisiones con alcance social y político, en las que predominan los intereses particulares y de grupo basados en criterios de competitividad y rentabilidad (31). Estos conflictos de valores se deben analizar desde una ética deontológica, a fin de otorgar legitimidad a la moral constitucional y limitar las consecuencias que tiene, en la práctica, el predomino de la moral de la economía de mercado aplicada en el sector de la salud.

\section{Dignidad humana y salud: derechos inherentes al ser humano}

Según los resultados encontrados, en el SGSSS colombiano no se asumen la dignidad humana y la salud como derechos inherentes al ser humano, sino como bienes de mercado. Si el fin primordial de los actores es la rentabilidad financiera por encima de la atención de las necesidades de salud de la po- blación, la dignidad humana pasa a un segundo plano y el individuo se convierte en un medio y no en un fin. Algunos autores, como Gañán (14) y Vargas y colaboradores (32), han calificado de rentista el SGSSS colombiano. Ya en 1999, Escobar afirmó que "la Ley 100 , de seguridad social en salud, tiene un enfoque basado en las teorías utilitaristas. Su valor básico es la utilidad y el mercado" (33). También la Defensoría del Pueblo se sumó a las críticas al señalar que "el nuevo modelo de atención en salud creado en Colombia por la Ley 100 convirtió el cuidado médico en un bien de mercado. La atención de la salud es ahora una industria..." (12).

Si bien la eficiencia es fundamental en cualquier proyecto donde se involucren recursos públicos, en la salud, como derecho fundamental, esta debe interpretarse en términos sociales y humanos, no meramente financieros. Al respecto, Maliandi afirma que “... en la base de una ética bien entendida tiene que estar, por ejemplo, la distinción que Kant establecía entre 'dignidad' y 'precio'. Lo que tiene precio puede ser cambiado por algo equivalente, mientras que nada equivalente hay para aquello que tiene dignidad. 'El necio', escribió Antonio Machado, 'confunde valor y precio'" (34). En la práctica del SGSSS prevalece la moral utilitarista auspiciada por el sistema económico neoliberal y se confunde el valor de la dignidad humana con el precio de los servicios.

\section{El profesional del sistema ¿autónomo o autómata?}

Para que una acción sea autónoma se debe ejecutar con libertad, conocimiento y sin coacción (35). En este sentido, la formación profesional debe hacer hincapié no solo en la formación técnica y científica, sino también en la educación moral y la reflexión ética, para poder tomar decisiones pertinentes, racionales, científicamente fundamentadas, justas y equitativas, y orientadas al bien de los pacientes. Además de los valores, que desempeñan un papel fundamental en la conducta humana (36), la autonomía garantiza que el médico pueda emitir su juicio profesional acerca del tratamiento, lo que constituye un derecho y una garantía para el paciente (37).

En este trabajo se observó que la autonomía del profesional de la salud se ve limitada por la relación laboral autorita- 
ria que restringe su capacidad de decisión. Sin embargo, una sólida educación moral y ética y una adecuada formación técnico-científica del profesional deben garantizarle la capacidad necesaria para identificar las presiones de una moral empresarial rentista y proteger su autonomía y los derechos de los pacientes. En términos de Freire (38), se requieren procesos de reflexión y educación que promuevan el fortalecimiento de una conciencia crítica.

\section{Los principios de honestidad, solidaridad, responsabilidad, cooperación y confianza}

Sin respeto a los principios de honestidad, solidaridad, responsabilidad, cooperación y confianza, la corrupción se apodera del sistema. La honestidad se pierde cuando el personal de salud supedita el bienestar del paciente a intereses personales o institucionales, ya sea mediante regalos o subvenciones; esto se facilita por las malas condiciones laborales, que hacen a los profesionales sanitarios más proclives a aceptar este tipo de presiones empresariales. Igualmente, el clientelismo político propicia la corrupción y los conflictos de intereses $(39,40)$ debido a los nexos clientelares entre quienes ocupan cargos en las instituciones estatales de salud con grupos económicos, propietarios de las aseguradoras y partidos políticos.

Al desviar los recursos públicos de salud destinados a la población más vulnerable para favorecer a particulares, la corrupción reproduce la inequidad. Investigaciones realizadas por instituciones de vigilancia y control han informado que en la Asociación Colombiana de Empresas de Medicina Integral y 14 aseguradoras privadas se detectaron graves situaciones de corrupción, desviación de recursos de salud, pactos de negación de servicios a pacientes, y altos e injustificados recobros al Ministerio de la Protección Social por servicios del plan obligatorio de salud (18). También se ha informado de casos de corrupción en el Ministerio de Salud para obtener mayores beneficios económicos $(18,41)$. Ante el predominio del interés lucrativo en el sistema, los actores pierden confianza entre sí, alejándose del objetivo de cuidar y mejorar la salud de la población.

El utilitarismo privilegia la libertad individualista sin asumir la responsabilidad. Sin embargo, desde una ética de responsabilidad se debe considerar el alcance de las acciones emprendidas en aras de preservar la vida humana y su dignidad, lo que implica señalar el bien a realizar y el mal a evitar (42). Según Camps, "las organizaciones son agentes morales, constructores de una cultura y un clima que influyen en el comportamiento de sus miembros" (43), por lo que se hace necesario también asumir una responsabilidad colectiva.

Los resultados encontrados resaltan la incompatibilidad entre la moral de la economía de mercado y los valores y principios constitucionales que deben regir el sistema de salud para proteger la vida y los derechos de la población; por ejemplo, se observó que la competencia va en contra de la solidaridad, mientras la rentabilidad y la eficiencia administrativa trabajan en contra de la dignidad, la responsabilidad, la autonomía y la calidad.

Este análisis reviste importancia no solamente para Colombia, sino también para otros países en desarrollo, dadas las directrices internacionales de reformas sanitarias que promueven la privatización, la economía de mercado y la reducción del Estado, como estrategias para mejorar el acceso, la calidad, la eficiencia, la efectividad, la equidad y la cobertura en el sector de la salud. Contrariamente, este modelo de mercado competitivo ha promovido el desarrollo de servicios curativos y ha desestimulado las acciones de promoción de la salud y prevención de las enfermedades, porque no son rentables a corto plazo. $(5,44)$. Al respecto, Sen plantea que es una violación del principio de justicia particularmente grave que algunas personas puedan no tener la oportunidad de alcanzar una buena salud debido a acuerdos sociales y no a una decisión personal de no preocuparse por la salud (45).

Los hallazgos de este estudio confirman lo encontrado por Basu y colaboradores (46) en una revisión sistemática sobre el desempeño de sistemas sanitarios públicos y privados en países de ingresos bajos y medios. En esa investigación se encontró que el sector privado violaba con mayor frecuencia los estándares médicos, generaba menor impacto en los pacientes y tenía una menor eficiencia, debido a las prescripciones innecesarias y perversas.

Los argumentos presentados invitan a reflexionar sobre los efectos negativos de la moral fundamentada en la economía de mercado que ha servido de base a las reformas de salud en Colombia y a buscar mecanismos para legitimar los principios y los valores constitucionales como garantes del derecho a la salud y la dignidad humana.

La metodología cualitativa empleada no permite generalizar los hallazgos y estos se circunscriben a la comprensión del tema de estudio en las ciudades analizadas. Tampoco se hace un análisis estadístico de las categorías estudiadas, como equidad, acceso y calidad, entre otras.

No obstante esas limitaciones, los resultados de esta investigación indican que en el SGSSS colombiano se presenta un conflicto entre la moral que patrocina el modelo de mercado y los valores y principios constitucionales, con un predominio - en la práctica- de la primera. El enfoque utilitarista e individualista predominante promueve el lucro individual y empresarial por encima del interés colectivo y favorece prácticas que van en contra de los derechos de las personas y la comunidad. Para la toma de decisiones en salud es necesario tener en cuenta, además de los elementos técnicos y científicos, los principios y valores que están enfrentados y reflexionar sobre ellos para su legitimación. La reflexión ética debe poner de manifiesto estas situaciones para generar procesos de legitimación de la axiología constitucional del Estado social de justicia, como garante del derecho a la salud y la dignidad humana.

Se debe promover el debate latinoamericano sobre la ética en los sistemas de salud para orientar las directrices de reforma de estos sistemas, cuyo fin debe ser garantizar la dignidad y los derechos humanos en la salud. También se debe fortalecer la formación constitucional y ética en los programas académicos de salud y otras áreas relacionadas con este sector - como derecho, economía, administración, ingeniería, entre otras- para garantizar que se respeten los derechos y la dignidad de las personas en el sector de la salud.

Agradecimientos. El contenido de este documento se deriva de la investigación sobre decisiones en salud en ambientes controlados por el Sistema General de Seguridad Social en Salud de Colombia, realizada entre 2007 y 2009 con el apoyo de la Universidad de Antioquia, la Universidad Industrial de Santander, 
el Instituto de Estudios del Ministerio Público de la Procuraduría General de la Nación y COLCIENCIAS. En ese estudio participaron, además de los autores de este artículo, Julián Vargas, Iván Felipe Muñoz, Juan José Acosta, Diego Sarasti, Yomaira Higuita, Argiro Berrío Castaño, Francisco Arcieri, Blanca Nubia Carva- jal, Flor de María Cáceres, José Albeiro Pulgarín, Margarita Quiroz, Beatriz Elena Londoño, Alejandro Arango, María Cristina Márquez, Natalia Velázquez y Diana Patricia Marín. Los resultados de esa investigación se incluyeron en el libro Dilemas en las decisiones en la atención en salud. Ética, derechos y debe- res constitucionales frente a la rentabilidad financiera, 2009. Se agradece al profesor Fernando Peñaranda por sus valiosos aportes en los temas relacionados con la moral y la ética.

\section{Conflictos de interés. Ninguno.}

\section{REFERENCIAS}

1. Pieper AM. Ética y moral. Barcelona: Editorial Crítica; 1991.

2. Berger P, Luckmann T. La construcción social de la realidad. Buenos Aires: Amorrortu Editores; 1968.

3. Tugendhat E. Lecciones de ética. Barcelona: Gedisa; 1997.

4. Cortina A. El mundo de los valores. Ética y educación. Bogotá: Editorial El Búho; 2009.

5. Homedes N, Ugalde A. Neoliberal reforms in health services in Latin America: a critical view from two case studies. Rev Panam Salud Publica. 2005;17(3):210-20.

6. Congreso de la República de Colombia. Ley 100 de 1993: por la cual se crea el sistema de seguridad social integral y se dictan otras disposiciones. Bogotá: El Congreso; 1993.

7. The World Bank. World development report. New York: Oxford University Press; 1993.

8. Granados RT, Gómez MC. La reforma de los sistemas de salud en Chile y Colombia: resultados y balance. Rev Salud Publica. 2000;2(2):97-120.

9. Restrepo J, Lopera J, Rodríguez S. Integración vertical en el sistema de salud colombiano: aproximaciones empíricas y análisis de doble marginalización. Borradores del CIE. 2005;15.

10. Colombia, Corte Constitucional. Sentencia T-571 de 1992. Bogotá: La Corte; 1992.

11. Echeverri O. Commoditization of health care services for development: the case of Colombia. Rev Panam Salud Publica. 2008;24(3): 210-16.

12. Colombia, Defensoría del Pueblo. Autonomía médica y su relación con la prestación de los servicios de salud. Bogotá: Defensoría del Pueblo; 2007.

13. Colombia, Defensoría del Pueblo. Evaluación de los servicios de salud que brindan las empresas promotoras de salud, 2005. Índice de satisfacción de usuarios -SUS-. Bogotá: Defensoría del Pueblo; 2005.

14. Gañán JL. Los muertos de la Ley 100: prevalencia de la libertad económica sobre el derecho fundamental a la salud, una razón de su ineficacia, caso del plan obligatorio de salud del régimen contributivo [tesis doctoral]. Bogotá: Universidad Externado de Colombia; 2010.

15. Peláez J. Durante el 2004, EPS, fuerte ascenso entre las 100 empresas más grandes de Colombia. Periódico El Pulso 2005 julio 10; sección Monitoreo:8 (col. 1).

16. Restrepo JH. Resultados financieros del seguro público de salud en Colombia, 1996-2005. Observatorio Seguridad Social. 2006;5(13):1-9.

17. Colombia, Defensoría del Pueblo. La tutela y el derecho a la salud 2011. Bogotá: Defensoría del Pueblo; 2011. Hallado en: http://www. defensoria.org.co/red/anexos/publicacio
nes/tutelaDerechosSalud2011a.pdf Acceso el 7 de agosto de 2012.

18. González LE. Catorce EPS serían multadas por hacer 'pacto' para negar servicios. Periódico El Tiempo 2011 abril 23:1.

19. Molina G, Ramírez A, Muñoz I, eds. Dilemas en las decisiones en la atención en salud. Ética, derechos y deberes constitucionales frente a la rentabilidad financiera en el sistema de salud colombiano. 2. ${ }^{a}$ ed. Bogotá: Editorial La Carreta; 2011.

20. Colombia, Corte Constitucional. Sentencia T-760 de 2008. Bogotá: La Corte; 2008.

21. Céspedes JE, Jaramillo I, Castaño RA. The impact of social security system reform on health services equity in Colombia. Cad Saude Publica. 2002;18(4):1003-24.

22. Vega R. Dilemas éticos contemporáneos en salud: el caso colombiano desde la perspectiva de la justicia social. Gerencia y Políticas de Salud (Bogotá). 2002;1(2):49-65.

23. Presidencia de la República. Constitución Política de Colombia 1991. Bogotá: La Presidencia; 1991. Hallado en: http:/ / web.presidencia. gov.co/constitucion/index.pdf Acceso el 13 de septiembre de 2012.

24. Otero M. Valores constitucionales. Introducción a la filosofía del derecho: axiología jurídica. 2. ${ }^{\text {a }}$ ed. España: Manuis Universitarios; 2004.

25. Rawls J. Teoría de la justicia. 2. a ed. México, D.F.: Fondo de Cultura Económica; 1995.

26. Denzin N, Lincoln Y. The landscape of qualitative research. $4 .^{\text {a }}$ ed. Beverly Hills, CA: Sage Publications; 2012.

27. Strauss A, Corbin J. Bases para la investigación cualitativa. Técnicas y procedimientos para desarrollar teoría fundamentada. Medellín: Universidad de Antioquia; 2002.

28. Gutiérrez Brito J. Grupo de discusión: ¿prolongación, variación o ruptura con el focus group? Cinta Moebio. 2011;41:105-22.

29. Paz Sandín E. Criterios de validez en la investigación cualitativa: de la objetividad a la solidaridad. Rev Investig Educativas. 2000;18(1): 223-42.

30. Moral SC. Criterios de validez en la investigación cualitativa actual. Rev Investig Educativas. 2006;24(1):147-64.

31. Guzmán J. El rol de la ética en la ciencia económica. Rev Etica Econ. 2005;823:11-27.

32. Vargas I, Vázquez ML, Jané E. Equidad y reformas de los sistemas de salud en Latinoamérica. Cad Saude Publica. 2002;18(4):927-37.

33. Escobar J. Ley 100 de seguridad social: implicaciones bioéticas. En: Escobar J, Da Costa M, Jácome S, Málaga H, Maldonado C, Montt J, et al., eds. Bioética y justicia sanitaria. 2. ${ }^{a}$ ed. Bogotá: El Bosque; 1999. Pp. 19.

34. Maliandi R. Ética: dilemas y controversias. Cuestiones éticas de la identidad, la globali- zación y la tecnología. Buenos Aires: Editorial Biblos; 2006.

35. Beauchamp T, Childress J. Principles of biomedical ethics. 6. ${ }^{\mathrm{a}}$ ed. New York: Oxford University Press; 2009.

36. Pedrajas M. La transformación ética de la racionalidad económica en Amartya Sen. Una recuperación de Adam Smith. Quaderns de filosofia i ciencia (Valencia). 2006;36:105-117.

37. Gómez R. Del "deber ser" y el "ser" del médico. Rev Colegio Jueces Fiscales Antioquia. 2002;22:5-11. Disponible en: http:// www.encolombia.com/medicina/pediatria/ pediatria39204-deldeber.htm Acceso el 13 de septiembre de 2008.

38. Freire P. Pedagogía de la autonomía: saberes necesarios para la práctica educativa. México D.F.: Siglo XXI; 2004.

39. Rotondo MT. Relaciones profesionales: conflicto de intereses. Rev Med Urug. 2006;22(2): 88-99.

40. Thompson DF. Understanding financial conflicts of interest. N Engl J Med. 1993;329(8): 573-6.

41. Legalizadas las primeras cinco capturas por fraude al sector salud [artículo de prensa en Internet]. El Colombiano 2011 mayo 3. Disponible en: http://www.elcolombiano. com/BancoConocimiento/P/por_fraude en_el_sector_salud fueron_legalizadas cinco_capturas/por_fraude_en_el_sector salud_fueron_legalizadas_cinco_capturas. asp? CodSeccion=211 Acceso el 3 de mayo de 2011.

42. Rizo R. Ética de la responsabilidad. En: Giusti M. Ciclo de conferencias: Paradigmas de la ética; 2006 Mayo 9-18; Lima: Pontificia Universidad Católica del Perú; 2006. Pp. 1-14.

43. Camps V. Una vida de calidad. Reflexiones sobre bioética. Barcelona: Ares y Mares; 2001.

44. Esteves R. The quest for equity in Latin America: a comparative analysis of the health care reforms in Brazil and Colombia. Int J Equity Health. 2012;11:6.

45. Sen A. ¿Por qué la equidad en salud? Rev Panam Salud Publica. 2002;11(5-6):302-9. Disponible en: http://www.scielosp.org/ scielo.php?pid=S1020-49892002000500005 \&script=sci_arttext Acceso el 4 de junio de 2012.

46. Basu S, Andrews J, Kishore S, Panjabi R, Stuckler D. Comparative performance of private and public healthcare systems in low and middle income countries: a systematic review. PloS Med. 2012;9(6):e1001244

Manuscrito recibido el 15 de diciembre de 2011. Aceptado para publicación, tras revisión, el 11 de enero de 2013. 
ABSTRACT Objective. To present the conflicting moral issues that arise in clinical and administrative decision-making processes in Colombia's General Health Social Security System (SGSSS).

Conflicting values in Methods. A study was conducted between 2007 and 2009 in six Colombian cities Colombia's health system: balancing the market economy and constitutional legislation, 2007-2009 (Barranquilla, Bogotá, Bucaramanga, Leticia, Medellín, and Pasto) using a theorybased qualitative methodology. A total of 179 in-depth interviews were held with physicians, nursing personnel, and administrators with broad experience in the health sector, as well as 10 focus groups representing users and leaders of community organizations involved in health. The interviews, which followed a predetermined script and used semistructured questions, gathered personal and professional information from the respondents.

Results. The health care decision-making process in Colombia is seen from two different moral perspectives: the rentier, or profit-making, motive, characterized by a neoliberal view of the market economy (the practical perspective), and the constitutional axiology of social democracy (the regulatory perspective). It was found that the utilitarian and individualistic motive predominates, in which individual and business profits are promoted over the collective interest, and this trend favors practices that undermine the rights of people and the community.

Conclusions. Predominance of a morality that views the Colombian SGSSS in terms of the market model generates conditions that go against the principles and values that are supposed to guide the health system as guarantor of the right to health and human dignity. Health decisions should take into account not only technical and scientific criteria but also the principles and values involved, and consideration should be given to safeguarding them.

Key words Health systems; morale; ethics; privatization; Colombia. 